\title{
OPEN Mycobacterium tuberculosis Rv3160c is a TetR-like transcriptional repressor that regulates expression of the putative oxygenase Rv3161c
}

\author{
Hasan Tükenmez ${ }^{1,2,3,4 凶}$, Souvik Sarkar ${ }^{1,3}$, Saber Anoosheh ${ }^{1,3}$, Anastasiia Kruchanova ${ }^{2,3}$, \\ Isabel Edström ${ }^{2,3}$, Gregory A. Harrison ${ }^{5}$, Christina L. Stallings ${ }^{5}$, Fredrik Almqvist ${ }^{1,3}$ \& \\ Christer Larsson ${ }^{2,3,6 \bowtie}$
}

Tuberculosis, caused by Mycobacterium tuberculosis (Mtb), is a major health threat listed among the top 10 causes of death worldwide. Treatment of multidrug-resistant $M t b$ requires use of additional second-line drugs that prolong the treatment process and result in higher death rates. Our team previously identified a 2-pyridone molecule (C10) that blocks tolerance to the first-line drug isoniazid at $\mathrm{C} 10$ concentrations that do not inhibit bacterial growth. Here, we discovered that the genes rv3160c and $r v 3161 \mathrm{c}$ are highly induced by $\mathrm{C} 10$, which led us to investigate them as potential targets. We show that Rv3160c acts as a TetR-like transcriptional repressor binding to a palindromic sequence located in the rv3161c promoter. We also demonstrate that C10 interacts with Rv3160c, inhibiting its binding to DNA. We deleted the rv3161c gene, coding for a putative oxygenase, to investigate its role in drug and stress sensitivity as well as $\mathrm{C} 10$ activity. This $\Delta r v 3161 \mathrm{c}$ strain was more tolerant to isoniazid and lysozyme than wild type $M t b$. However, this tolerance could still be blocked by $\mathrm{C} 10$, suggesting that C10 functions independently of Rv3161c to influence isoniazid and lysozyme sensitivity.

Tuberculosis (TB) caused by Mycobacterium tuberculosis $(M t b)$ is still a major infectious disease worldwide ${ }^{1}$. In 2018, an estimated 10 million people fell ill with TB and 1.5 million died globally ${ }^{1}$. The fight against TB is exacerbated by the emergence of multidrug-resistant strains (MDR-TB) resistant to at least two of the first-line drugs to treat $\mathrm{TB}$, isoniazid (INH) and rifampicin ${ }^{1}$. These infections require additional second-line drugs and longer duration of treatment where the treatment success rate drops substantially ${ }^{1}$. Over the last 50 years, only three new TB drugs have been developed and approved for use against TB: bedaquiline, delamanid and most recently, pretomanid. However, there are already several cases reported with TB resistant to both bedaquiline and delamanid ${ }^{2-5}$. Therefore, there is still a desperate need to develop new strategies to target drug-resistant $M t b$.

Previously, our team described a thiazolo ring-fused 2-pyridone (C10) with the ability to increase sensitivity to oxidative stress, acid stress and the first-line antibiotic $\mathrm{INH}^{6}$. $\mathrm{C} 10$ was found to potentiate the bactericidal activity of INH and prevent the occurrence of INH-resistant mutants ${ }^{6}$. Moreover, INH-resistant katG mutants were re-sensitized to INH in the presence of C10, demonstrating that INH resistance can be circumvented ${ }^{6}$. We also showed that $\mathrm{C} 10$ inhibited respiration in $M t b$, and previous reports have shown that both inhibition and stimulation of $M t b$ respiration can potentiate bactericidal activity of $\mathrm{INH}^{7,8}$. However, the link between $\mathrm{C} 10$ effects on respiration and C10 effects on INH sensitivity were not elucidated. The C10 target(s) in $M t b$ as well as molecular mechanism of the INH potentiation is unknown.

Here, we discovered the rv3160c-rv3161c operon to be induced by $\mathrm{C} 10 \mathrm{in} \mathrm{Mtb}$. We found that C10 inhibits DNA-binding activity of Rv3160c, a putative TetR-like transcription factor, and results in increased expression of Rv3161c, a protein of unknown function related to oxygenases found in other bacteria. When knocked out,

\footnotetext{
${ }^{1}$ Department of Chemistry, Umeå University, 90187 Umeå, Sweden. ${ }^{2}$ Department of Molecular Biology, Umeå University, 90187 Umeå, Sweden. ${ }^{3}$ Umeå Centre for Microbial Research, Umeå University, 90187 Umeå, Sweden. ${ }^{4}$ Molecular Infection Medicine, Sweden (MIMS), Umeå University, 90187 Umeå, Sweden. ${ }^{5}$ Department of Molecular Microbiology, Washington University School of Medicine, St. Louis, MO 63110, USA. ${ }^{6}$ Present address: Holmsund, Sweden. ${ }^{凶}$ email: hasan.tukenmez@umu.se; christer.larsson1975@gmail.com
} 
Expression of the rv3160c-rv3161c operon

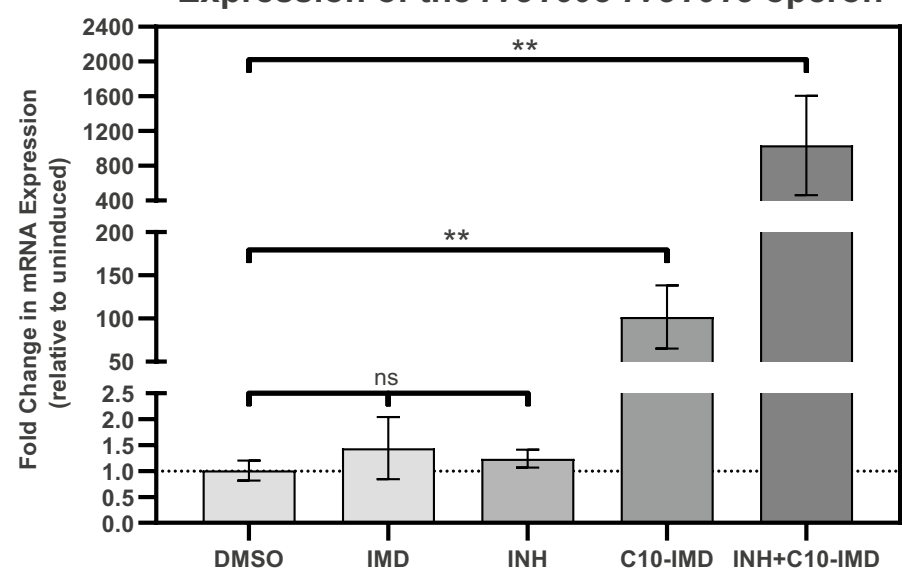

Figure 1. Expression of the $r v 3160 c-r v 3161 c$ operon is significantly induced by C10-IMD. Mtb Erdman WT was cultivated in Sauton's medium at $37^{\circ} \mathrm{C}$ until its $\mathrm{OD}_{600}$ reached 0.3 . The cells were then exposed to $0.1 \%$ DMSO, $0.1 \mu \mathrm{g} / \mathrm{ml} \mathrm{INH}$ and $25 \mu \mathrm{M}$ C10-IMD separately or in combination for $48 \mathrm{~h}$ at $37^{\circ} \mathrm{C}$. Differences in expression of the $r v 3160 c-r v 3161 c$ operon were determined by qRT-PCR analysis performed by targeting $r v 3161 c$ coding region. Expression of the sigA gene was determined by qRT-PCR analysis and used as housekeeping control. Relative fold-change in $r v 3160 c-r v 3161 c$ operon expression was calculated for each biological replicate by the Livak $\left(2^{-\Delta \Delta C t}\right)$ method. Bar graphs were plotted based on average and standard deviation obtained from three independent biological replicates and statistical analysis comparing to the DMSO control was determined by one-tail $t$-test that was performed using GraphPad Prism version 8.4.3 for Windows, GraphPad Software, San Diego, California USA, www.graphpad.com $\left({ }^{* *} p<0.01\right)$.

$\Delta r v 3161 c$ mutants became more tolerant to both INH and lysozyme, indicating that the Rv3161c plays an active role in both INH potentiation and maintenance of cell wall integrity. However, the $\Delta r v 3161 c$ mutants can still be sensitized to both INH and lysozyme by C10, excluding the potential involvement of $r v 3160 c-r v 3161 c$ in the C10 effect.

\section{Results}

Expression of the $r v 3160 c-r v 3161 c$ operon is upregulated in response to $\mathrm{C} 10$. In our previous study, the small molecule $\mathrm{C} 10$ was discovered to reduce tolerance to oxidative stress, acid stress and to the first line antibiotic INH in $M t b^{6}$. Furthermore, C10 could restore INH sensitivity in otherwise INH-resistant kat $G$ mutants ${ }^{6}$. C10 also perturbed respiratory homeostasis, but its mechanism of action is unclear ${ }^{6}$.

In order to identify the target(s) of $\mathrm{C} 10$ and discover the mechanism behind its INH potentiation, we revisited our previous RNA-seq data and looked closer at the top genes induced by C10. The $r v 3160 c$ and $r v 3161 c$ genes, positioned within a proposed two-gene operon ${ }^{9,10}$, were among the top 5 genes upregulated by $\mathrm{C} 10$ under hypoxic growth conditions (13-fold and 12 -fold, respectively $)^{6}$. These genes particularly caught our attention, as they have been shown to be induced by triclosan, an antibacterial that inhibits InhA, resulting in deficient mycolic acid synthesis in $M t b$, similar to $\mathrm{INH}^{11,12}$.

According to the RNA-seq results, upregulation of the $r v 3160 c-r v 3161 c$ operon by $\mathrm{C} 10$ was observed under hypoxic growth conditions but not under aerated conditions. To investigate this further, we performed quantitative real-time PCR (qRT-PCR) analysis of the $r v 3160 c-r v 3161 c$ operon in response to C10 molecule under aerobic conditions. To improve solubility of C10, we used a C10-imidazole salt (C10-IMD) instead of C10 during this study (Fig. S1) ${ }^{6}$. qRT-PCR data showed that expression of the $r v 3160 c$-rv3161c operon increases $102 \pm 32$-fold under aerated growth conditions upon exposure to $25 \mu \mathrm{M}$ C10-IMD for $48 \mathrm{~h}$ (Fig. 1). Furthermore, expression of this operon was induced even further, $1035 \pm 571$-fold upon exposure to a combination of $25 \mu \mathrm{M} \mathrm{C10-IMD}$ and $0.1 \mu \mathrm{g} / \mathrm{ml} \mathrm{INH}$ for $48 \mathrm{~h}$ (Fig. 1). There were no significant changes in expression of the operon upon exposure to either $0.1 \mu \mathrm{g} / \mathrm{ml}$ INH or $25 \mu \mathrm{M}$ IMD alone for $48 \mathrm{~h}$ (Fig. 1). These findings suggest that INH and C10-IMD have a synergistic role in the induction of the $r v 3160 c-r v 3161 c$ operon. Based on this, we decided to investigate the $r v 3160 c-r v 3161 c$ operon further.

Rv3160c is a TetR-like transcription repressor of the $r v 3160 c-r v 3161 c$ operon. The $r v 3160 c$ gene encodes a putative TetR family transcriptional regulator (TFTR) and is uniquely found in mycobacteria causing $\mathrm{TB}^{13}$. Mycobacterial genomes are highly enriched with several TFTRs, of which the majority act as repressors of gene expression ${ }^{13}$. Even though Rv3160c has been suggested to be a TFTR based on sequence domain similarities to other TFTRs, its function and possible target(s) have not been described. The majority of the TetR-like transcriptional repressors are known to regulate their neighboring genes and often to be co-transcribed with their regulatory targets ${ }^{13}$. Several previous studies have suggested that the $r v 3160 c$ and the $r v 3161 c$ genes are located within the same operon based on their correlated gene expression in response to various environmental factors $^{11,14-19}$. However, a genome wide study suggested the $r v 3160 c$ gene to have its own independent transcrip- 
A)

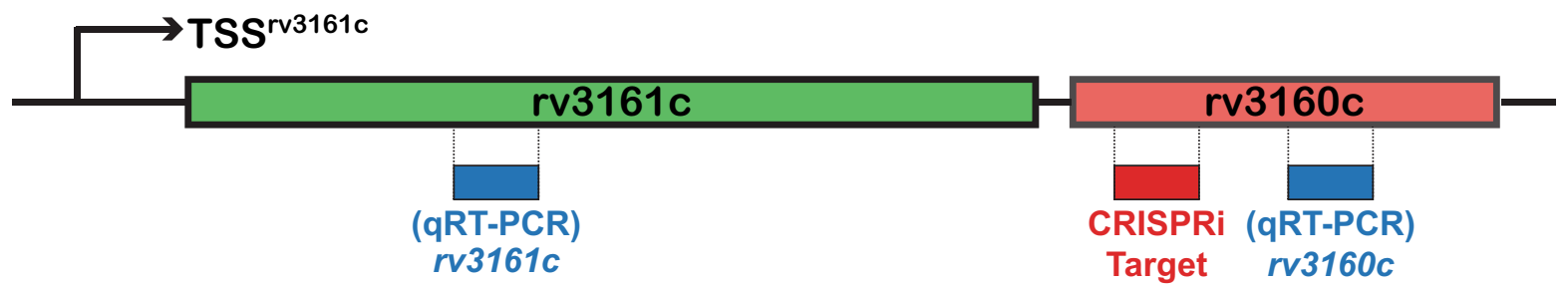

B)

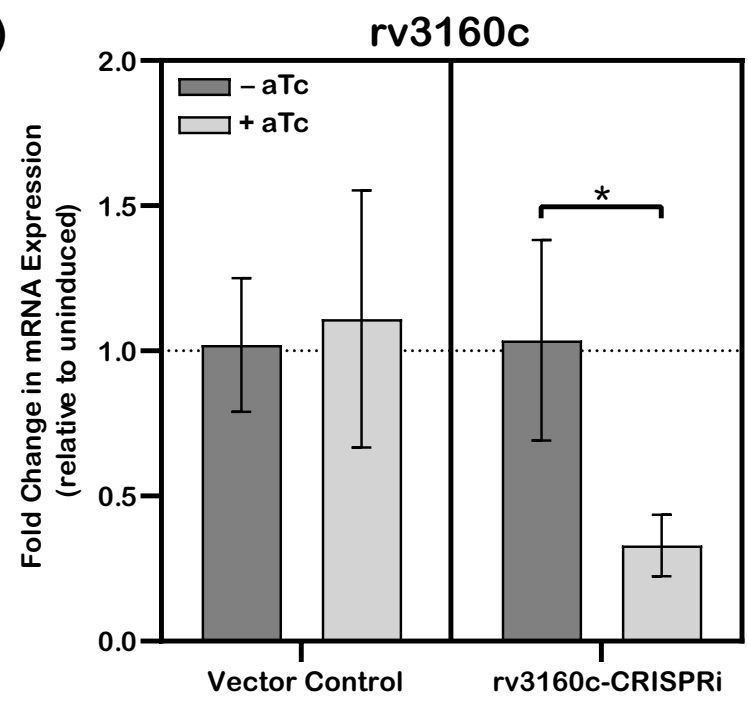

C)

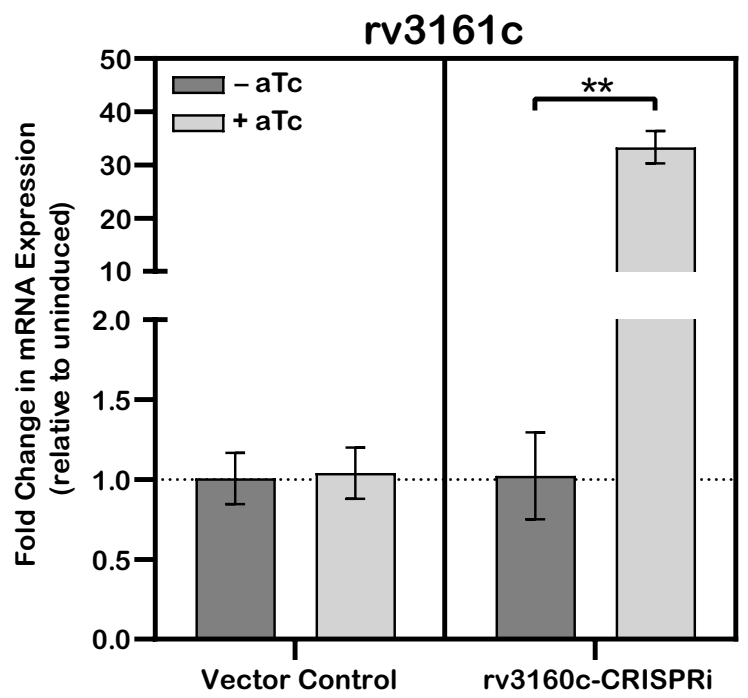

Figure 2. Rv3160c acts as transcriptional repressor of the $r v 3160 c-r v 3161 c$ operon. (A) Schematic representation of the $r v 3160 c-r v 3161 c$ operon and the relative positions of the targets for CRISPRi and qRTPCR. (B,C) qRT-PCR analysis of $r v 3160 c$ and $r v 3161 c$ mRNA expression in Mtb Erdman WT strain harboring either vector control (pJR965) or the rv3160c-CRISPRi (pSA253) construct in absence (-) or presence (+) of $100 \mathrm{ng} / \mathrm{ml}$ aTc. sigA mRNA expression was used as control and relative fold change in $r v 3160 \mathrm{c}$ and $r v 3161 \mathrm{c}$ mRNA expression were calculated for each biological replicate by the Livak $\left(2^{-\Delta \Delta \mathrm{Ct}}\right)$ method. Average value of 3 technical replicates was used for each biological replicate. Bar graphs were plotted based on average and standard deviation obtained from three independent biological replicates and statistical analysis was determined by one-tail $t$-test that was performed using GraphPad Prism version 8.4.3 for Windows, GraphPad Software, San Diego, California USA, www.graphpad.com $\left({ }^{*} p<0.05,{ }^{* *} p<0.01\right)$.

tional start site (TSS) starting at 406 bp upstream of the $r v 3160 c$ open reading frame (ORF), overlapping with the $r v 3161 c \mathrm{ORF}^{20}$. To determine which promoter region was responsible for the transcription of $r v 3160 c$ in our conditions, we measured the expression levels of the $r v 3160 c$ gene while targeting the region between the $r v 3160 c-r v 3161 c$ operon TSS and the recently proposed independent TSS of the $r v 3160 c$ gene with a CRISPRi construct (Fig. S2). We found that the expression of the $r v 3160 c$ gene was significantly reduced suggesting that $r v 3160 c$ is mainly co-transcribed with the $r v 3161 c$ gene (Fig. S2). However, the existence of additional TSSs, perhaps of importance in response to other conditions, cannot be excluded based on this data.

We designed an experiment to test whether expression of the neighboring $r v 3161 c$ gene was regulated by Rv3160c (Fig. 2A). We reduced expression of $r v 3160 c$ gene ( threefold) by $r v 3160 c$-CRISPRi (Fig. 2B), which resulted in a $33 \pm 3$-fold increased expression of the $r v 3161 c$ gene (Fig. $2 \mathrm{C}$ ). This data shows that decreasing $r v 3160 c$ expression results in an increase in $r v 3161 c$ expression, although it does not show whether this occurs through direct regulation or not.

TFTRs typically bind to palindromic motifs as dimers, thus we searched for such motifs in the upstream sequence of the $r v 3160 c-r v 3161 c$ operon. We identified a stretch of $50 \mathrm{bp}$ containing 12-bp and 8-bp inverted repeats starting at the -29 bp upstream of the operon (Fig. 3A). We confirmed that Rv3160c binds to a $142 \mathrm{bp}$ DNA fragment containing these palindromic motifs, resulting in a DNA shift using the electrophoretic mobility shift assay (EMSA) (Fig. S3). We then digested the $142 \mathrm{bp}$ DNA fragment with different restriction enzymes to narrow down the region that is targeted by Rv3160c (Fig. S3). We found that that presence of both inverted repeats as well as the -10 region were necessary for an efficient interaction, thus we decided to use 86 bp DNA fragment containing both these palindromic motifs for the remaining EMSAs (Fig. 3B). The binding of Rv3160c to these palindromes would block access of the RNAP to the - 10 sigma factor binding site and repress transcription of the rv3161c. To test if the palindromic motifs were important for Rv3160c binding, we performed EMSAs with modified DNA fragments. We used a higher concentration of Rv3160c (100 pmol) in these assays compared to those presented in Fig. 3 ( $10 \mathrm{pmol}$ ) to ensure the protein was in excess to detect lower affinity binding events. In these experiments, we observed that our DNA dye bound to purified Rv3160c (Fig. S4), obscuring the shifted 
A)

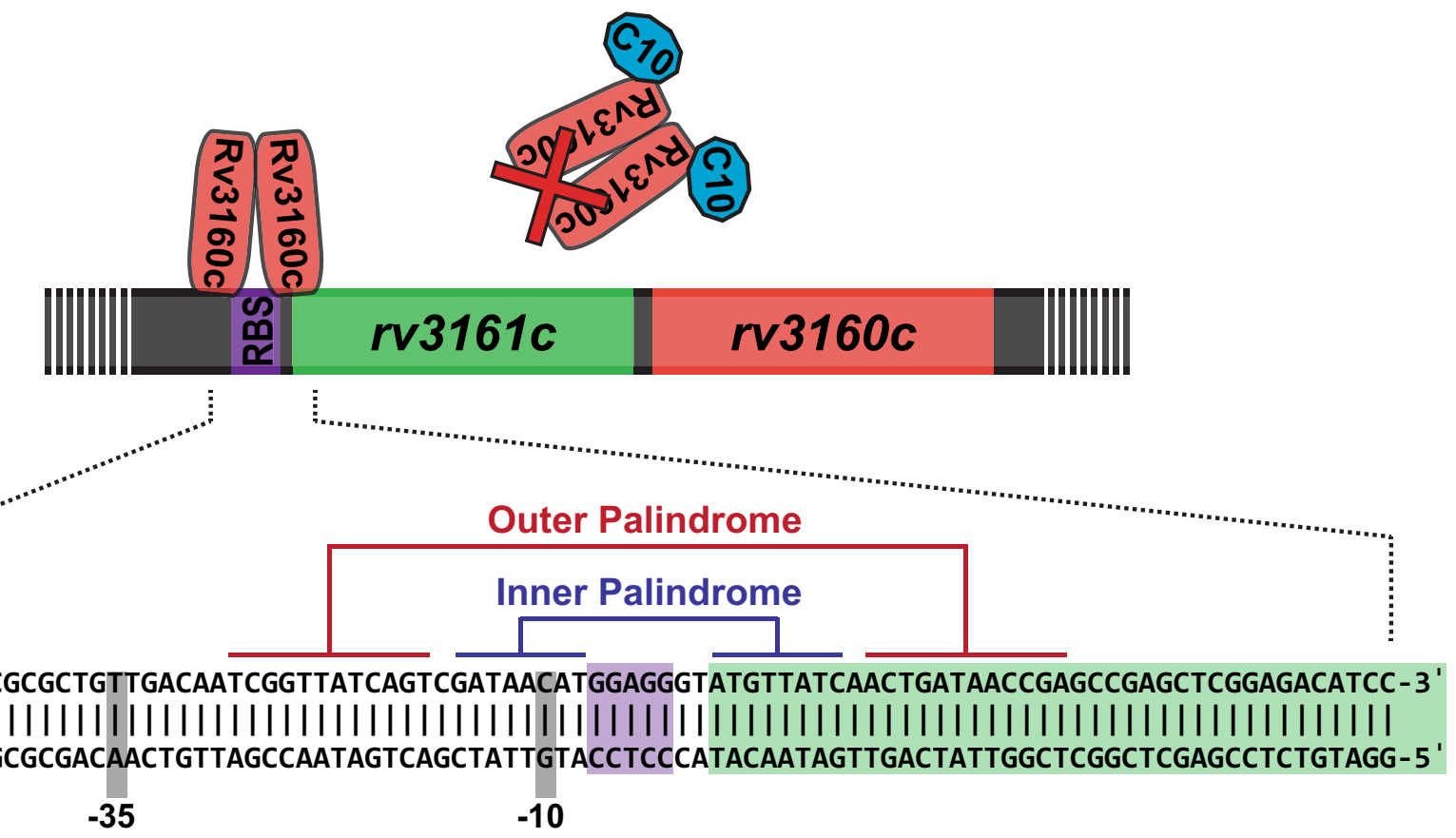

B)

Upstream of $r v 3161 c$

(86 bp: $-46 \ldots 40$ )

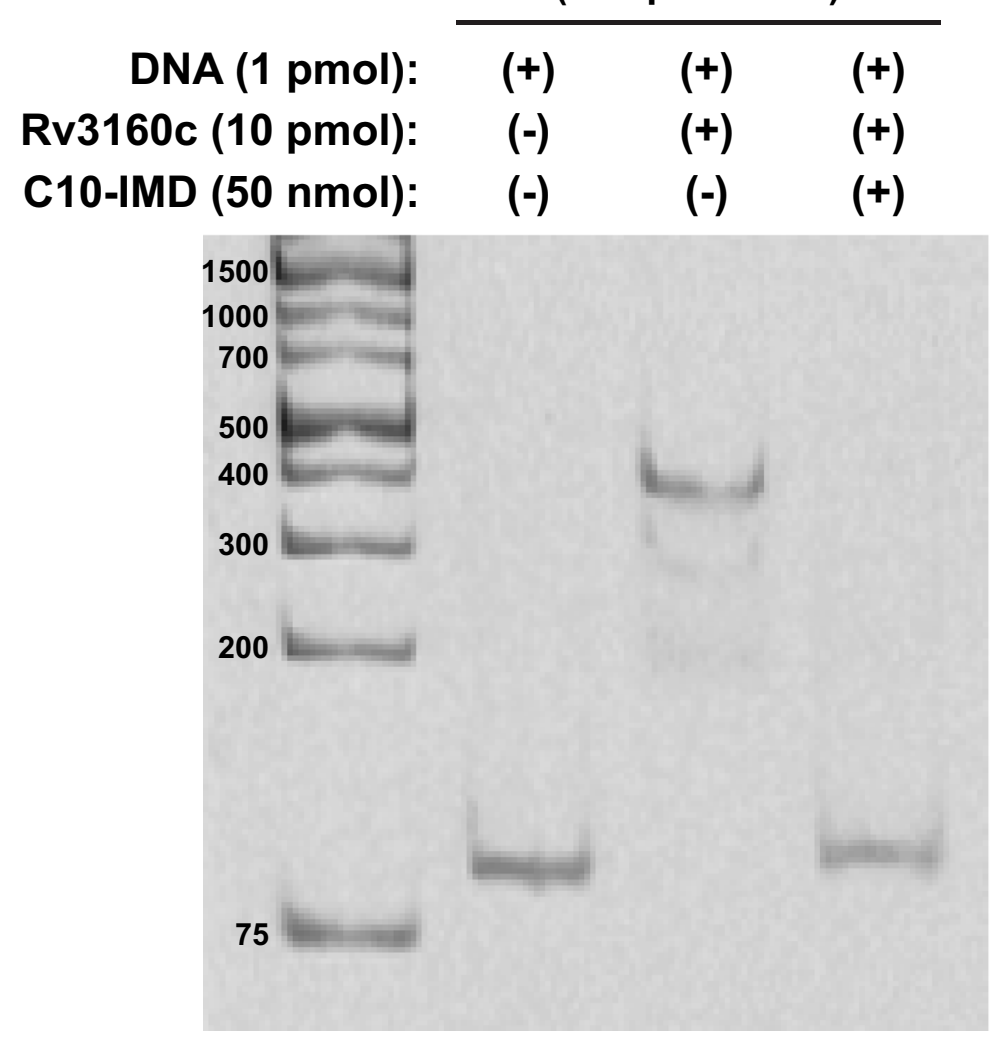

Upstream of cfp10 (204 bp: -152...50)

$(+) \quad(+)$

$(-) \quad(+)$

$(-)$

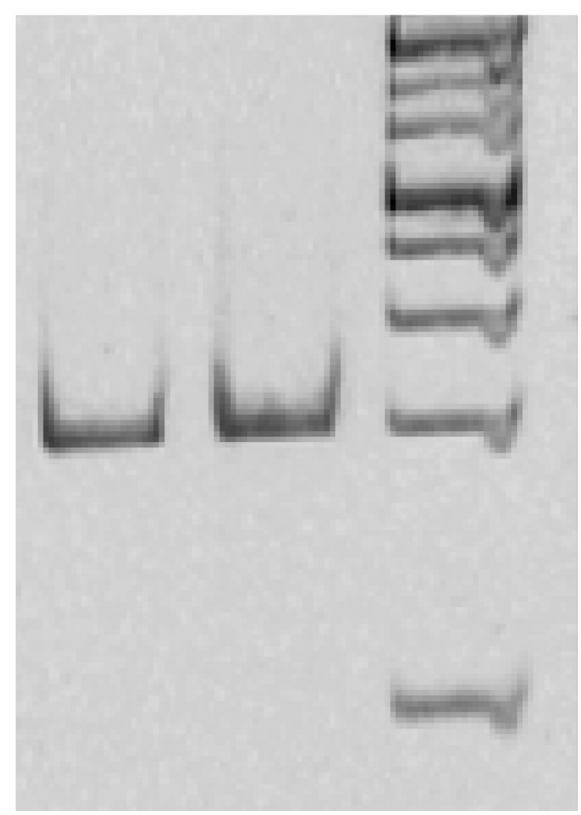

Figure 3. Binding of Rv3160c to the rv3160c-rv3161c operon is blocked by C10-IMD. (A) Schematic depicting model of the Rv3160c dimer binding to the palindromic sequence present in the rv3161c upstream fragment. (B) EMSA assay using an 86 bp rv3161c upstream fragment (1 pmol; lanes 1-3) and a 204 bp cfp10 upstream fragment as negative control fragment ( 1 pmol; lanes 4-5). Lane 1 and 5: no protein added. Lanes 2, 3 and 5: binding reaction with 10 pmol Rv3160c. Lane 3: $50 \mathrm{nmol} \mathrm{C10-IMD.} \mathrm{DNA} \mathrm{Ladder:} \mathrm{Thermo} \mathrm{Scientific} \mathrm{GeneRuler}$ $1 \mathrm{~kb}$ Plus (SM1331). 
DNA band. Nevertheless, this interaction would not interfere with the migration of the band in the acrylamide gels as staining took place after the gel runs were completed. We evaluated Rv3160c binding by quantifying the amount of unbound DNA in the presence of Rv3160c as a percentage of the unbound DNA in the absence of $\mathrm{Rv} 3160 \mathrm{c}$ for each DNA construct. To test if the inner palindrome is important for Rv3160c binding, we shuffled the 8 bp inverted repeats in the inner palindrome, without eliminating the palindrome structure or base composition (Fig. S5A). Rv3160c showed decreased binding to the fragment harboring a shuffled inner palindrome, leaving $83 \%$ of the DNA unbound, as compared to the unmodified fragment, where only $8 \%$ of the DNA remains unbound by Rv3160c (Fig. S5B). We then incubated Rv3160c with DNA fragments harboring a shuffled outer palindrome or harboring both shuffled inner and outer palindromes and found that nearly all of the DNA remained unbound in the presence of Rv3160c (Fig. S5). Furthermore, by modifying the two non-matching nucleotides located between the inner and outer palindromes to create a single $21 \mathrm{bp}$ inverted repeat, we found that Rv3160c was still able to bind most of the DNA fragment, leaving 37\% of the DNA unbound (Fig. S5A). These findings demonstrate that the ability of Rv3160c to bind the sequence upstream of $r v 3161 c$ is dependent on the sequence of both inverted repeats.

C10-IMD inhibits the interaction between Rv3160c and the rv3161c upstream target sequence. As mentioned above, expression of the $r v 3160 c-r v 3161 c$ operon is induced by C10-IMD. Thus, we hypothesized that C10-IMD might interfere with Rv3160c function. Indeed, we found that Rv3160c was no longer capable of interacting with the sequence upstream of $r v 3161 c$ in the presence of C10-IMD in the EMSA (Fig. 3B). To investigate whether C10-IMD interacted directly with Rv3160c, we analyzed the effect of C10-IMD on Rv3160c tryptophan fluorescence emission. There are 3 tryptophan residues available in Rv3160c and the binding interaction between C10-IMD and Rv3160c was studied using tryptophan fluorescence. The presumption is that if C10-IMD binds Rv3160c and some of the tryptophan residues are in close proximity to the C10-IMD binding site, compound binding might affect the intrinsic fluorescence of Rv3160c. Rv3160c was titrated with increasing concentrations of C10-IMD and relative fluorescence intensity was found to decrease in a concentration-dependent manner (Fig. 4A). The fluorescence quenching was further analyzed by Stern-Volmer equation (Eq. 1, see "Methods" section) to determine the Stern-Volmer quenching constant $\left(\mathrm{K}_{\mathrm{sv}}\right)$ (Fig. 4B), which signifies the equilibrium binding constant for complex formation in the light-induced electronic excited state resulting in the quenching of intrinsic fluorescence of tryptophan. In this study, the Stern-Volmer plot analyzes the type of fluorescence quenching of tryptophan residues in Rv3160c by plotting relative emission intensity against C10-IMD concentration. In the case of Rv3160c, a tryptophan might be buried in the protein, whose fluorescence quenching depends on the microenvironment, solvent accessibility, and binding of C10IMD. Overall, the tryptophan fluorescence quenching is a combined contribution of the microenvironment of all tryptophan residues in the presence of C10-IMD. If tryptophan residues are in close proximity of C10-IMD binding site, C10-IMD binding leads to fluorescence quenching of tryptophan while the fluorescence of other tryptophan residues that are not involved in C10-IMD binding would not be quenched. The Stern-Volmer plot, therefore, helps to understand the binding process leading to quenching phenomena ${ }^{21}$. To calculate the binding dissociation constant $\left(\mathrm{K}_{\mathrm{D}}\right)$ and the number of binding site $(\mathrm{n})$, the logarithmic Eq. (2) (see "Methods" section) was used. The $\log \left(\mathrm{K}_{\mathrm{b}}\right)\left(\mathrm{K}_{\mathrm{b}}\right.$ denotes binding association constant) value had been determined from the intercept of the plot, shown in Fig. $4 \mathrm{C}$. The binding dissociation constant $\left(\mathrm{K}_{\mathrm{D}}\right)$ was found to be $44.51 \pm 0.85 \mu \mathrm{M}$ using Eq. (3). The number of binding sites for the C10-IMD in Rv3160c was found to be $0.94 \pm 0.09$ suggesting one binding site of C10-IMD in Rv3160c.

TFTRs usually consist of two domains: one signal-receiving and the other one DNA-binding. Interactions of TFTRs with their target DNAs often lead to structural changes, especially in the DNA-binding domain ${ }^{22-25}$. Similarly, specific molecules can bind to the regulatory domain of TFTRs, resulting in conformational changes that make TFTRs incapable of binding their DNA targets ${ }^{26,27}$. According to our EMSA experiments, Rv3160c cannot bind to its DNA target upon addition of C10-IMD. Thus, we investigated whether interaction with C10IMD results in conformational changes in Rv3160c using circular dichroism (CD) spectroscopy. We discovered that the ellipticity of Rv3160c was reduced upon addition of its DNA target, indicating changes in the secondary structure (Fig. 4D). In contrast, no change in the ellipticity of Rv3160c was observed in the presence of C10-IMD (Fig. 4E), indicating that C10-IMD did not alter the secondary structure of Rv3160c in the absence of DNA. There is a slight change in the ellipticity in the presence of C10-IMD approximately at $208 \mathrm{~nm}$ in Fig. $4 \mathrm{E}$ does not have much impact on overall secondary structure of Rv3160c.

Deletion of $r v 3161 c$ results in increased sensitivity to INH and lysozyme. C10-IMD directly binds Rv3160c, a TetR-like transcriptional repressor, which could be leading to the increased expression of the $r v 3160 c-r v 3161 c$ operon in the presence of C10-IMD. We hypothesized that INH potentiation by C10-IMD might be regulated through the cellular concentration of Rv3161c, a putative oxygenase. To test this, we used recombineering to generate a $r v 3161 \mathrm{c}$ knock-out strain and tested its sensitivity to INH. Both wild type and $\Delta r v 3161 c$ strains were incubated for 2 weeks at $37^{\circ} \mathrm{C}$ with varying concentrations of INH in U-bottomed 96-well plates. After 2-weeks incubation, we scored the pellet formations in each well as a semi-quantitative indication of bacterial growth and spotted them on $7 \mathrm{H} 10$ plates followed by additional 3-weeks of incubation at $37^{\circ} \mathrm{C}$ to assess the presence of viable bacteria. We discovered that the $\Delta r v 3161 c$ strain was more resistant to INH in comparison to wild type, as it could survive up to double the INH concentration $(0.016 \mu \mathrm{g} / \mathrm{ml})$ (Fig. 5). We also observed that addition of $5 \mu \mathrm{M}$ C10-IMD potentiated INH sensitivity in both wild type and $\Delta r v 3161 c$ strains, demonstrating that the effect of C10-IMD on INH potentiation occurs independently of Rv3161c (Fig. 5).

Previous studies show that expression of the $r v 3160 c-r v 3161 c$ operon is upregulated in the lipid-rich environments $^{18}$ and upon treatment with cell wall synthesis inhibitors ${ }^{11,17,19}$, raising the question of whether 
A)

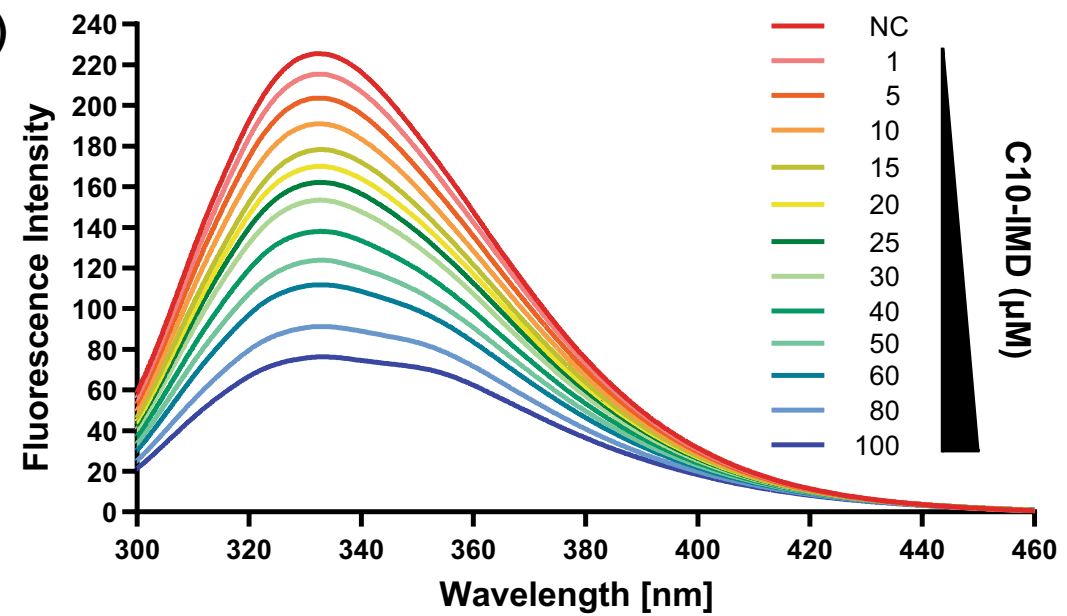

B)

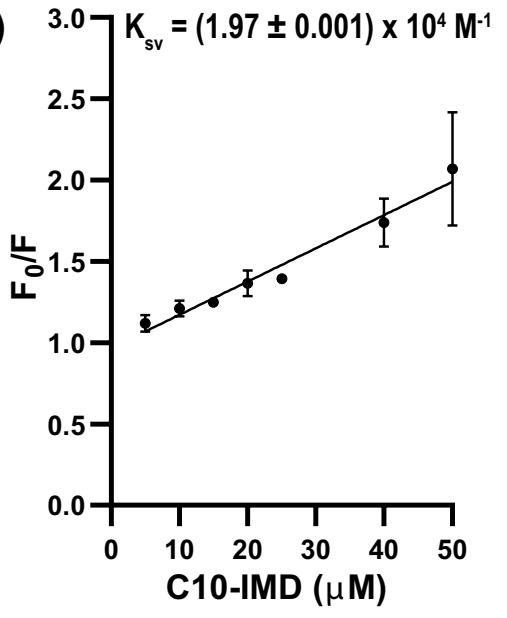

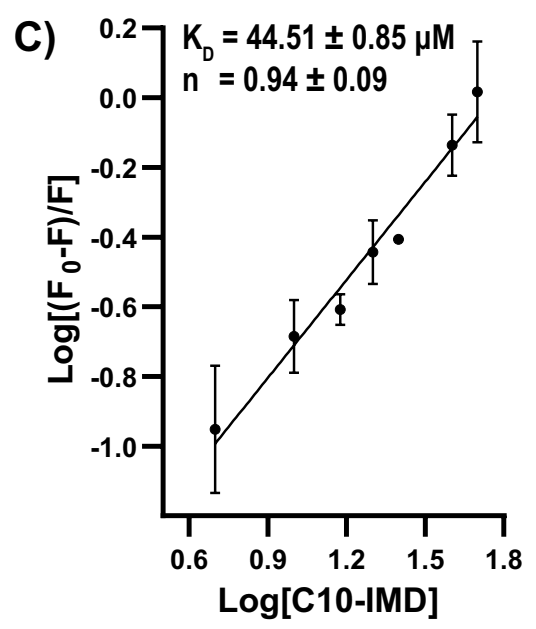

D)

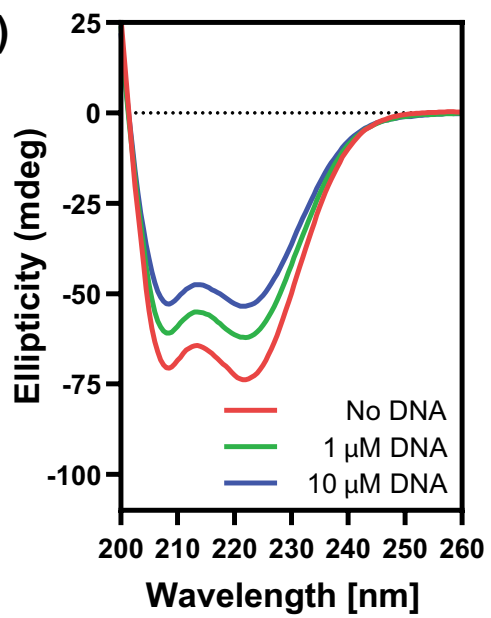

E)

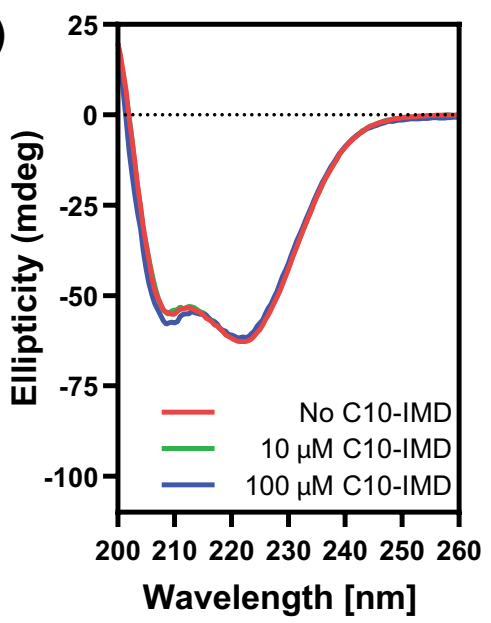

Figure 4. C10-IMD binds to a single site in Rv3160c and the interaction does not cause changes in the secondary structure of the protein. (A) Fluorescence quenching spectra of Rv3160c. The $10 \mu \mathrm{M}$ Rv3160c in PBS solution is excited and quenching of fluorescence is recorded in the presence of varying concentrations of C10IMD $(1-100 \mu \mathrm{M})$. (B) Stern-Volmer plot of decrease in mean fluorescence intensity of Rv3160c in the presence of varying concentrations of C10-IMD derived from three independent experiments. The dynamic quenching rate constant $\mathrm{K}_{\mathrm{sv}}$ is evaluated from the slope of the line. (C) Logarithmic plot of mean relative fluorescence quenching of Rv3160c derived from three independent experiments against logarithmic concentrations of C10-IMD. $K_{D}$ is calculated from the intersection of the line with the y axis and the number of binding sites (n) from the slope of the line. (D) CD spectra of Rv3160c in the presence of $1 \mu \mathrm{M}$ or $10 \mu \mathrm{M} 86 \mathrm{bp}$ rv3161c upstream fragment (DNA) (Fig. 2C). (E) CD spectra of Rv3160c in the presence of $10 \mu \mathrm{M}$ or $100 \mu \mathrm{M}$ C10-IMD. Error bars in $(\mathbf{B})$ and $(\mathbf{C})$ indicate standard deviation.

these genes play a role in cell wall integrity. We set up a similar growth and survival assay to test the cell wall integrity of both wild type and $\Delta r v 3161 \mathrm{c}$ strains by incubating them in the presence of varying concentrations of lysozyme, an antimicrobial enzyme that cleaves the peptidoglycan component of the bacterial cell wall. The $\Delta r v 3161 c$ strain survived exposure up to double the lysozyme concentration $(62.5 \mu \mathrm{g} / \mathrm{ml})$ compared to the wild type strain (Fig. 5). In addition, we found that both the wild type and $\Delta r v 3161 \mathrm{c}$ strains were more sensitive to lysozyme in the presence of $5 \mu \mathrm{M} \mathrm{C10-IMD} \mathrm{(Fig.} \mathrm{5).}$

\section{Discussion}

Expression of the $r v 3160 c-r v 3161 c$ operon has been reported to be induced by various anti-microbial agents such as triclosan $^{11,19}$, thioridazine ${ }^{16,17,19}$, SRI $\# 967^{15}$, SRI $\# 9190^{15}$ and chlorpromazine ${ }^{19}$. Rv3161c encodes a putative oxygenase that shares high amino acid sequence identity (32-62\%) with other Rieske oxygenases found in several organisms. Rieske oxygenases are known to be involved in the degradation of arenes, therefore it has been suggested that Rv3161 c might hydroxylate the benzene ring structures found in these anti-microbial agents resulting in detoxification ${ }^{11,15,17}$. However, neither deletion nor overexpression of the $r v 3161 c$ gene influenced the minimal inhibitory concentration (MIC) of triclosan or other anti-microbial agents ${ }^{28}$. Therefore, upregulation of $r v 3161 c$ expression was suggested to be a failed attempt of the bacteria to counteract these drugs ${ }^{28}$. In another study, mutations in the $r v 3161 \mathrm{c}$ gene were found to cause resistance against the 2,4-diaminoquinazoline (DAQ) 
A)

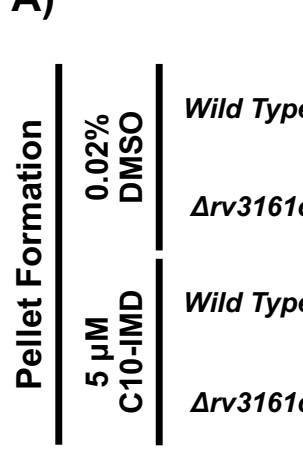

B)

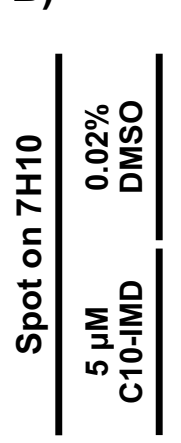

Isoniazid $(\mu \mathrm{g} / \mathrm{ml})$

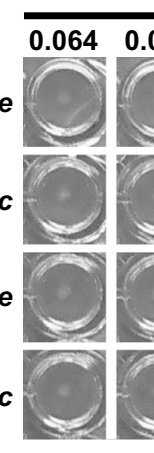

$0.016 \quad 0.008 \quad 0.004 \quad 0$

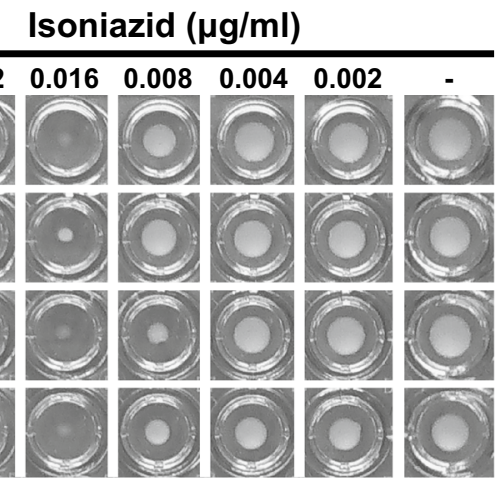

Isoniazid $(\mu \mathrm{g} / \mathrm{ml})$

$0.016 \quad 0.008 \quad 0.004 \quad 0$

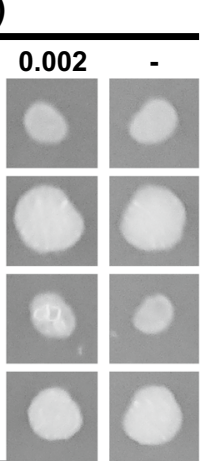

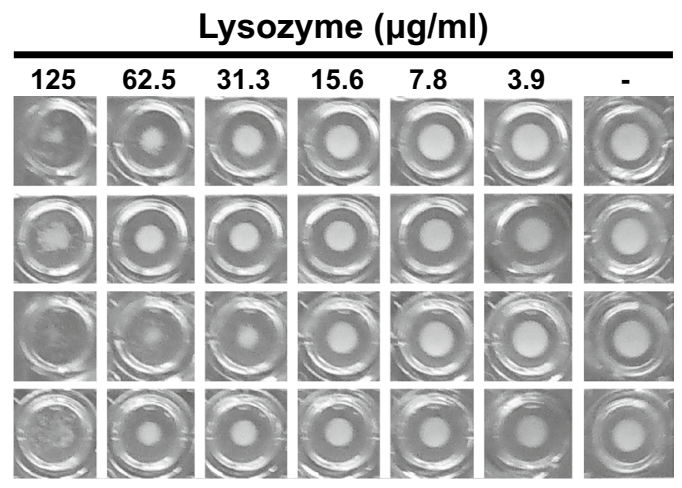

Lysozyme $(\mu \mathrm{g} / \mathrm{ml})$

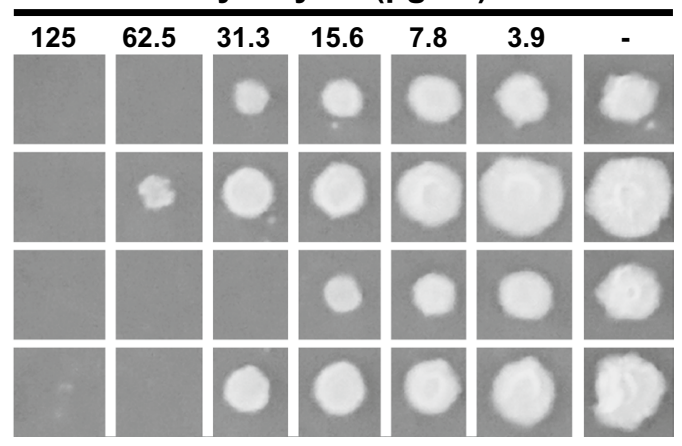

Figure 5. Deletion of the $r v 3161 c$ gene results in higher resistance to isoniazid and lysozyme. (A) Representative images showing effect of INH and lysozyme on bacterial growth scored by pellet formation. $M t b$ Erdman WT and $\Delta r v 3161 c$ strains were incubated 2 weeks in Sauton's medium with $0.02 \%$ DMSO or $5 \mu \mathrm{M}$ C10-IMD in addition to various concentration of INH (left; 0.002-0.064 $\mu \mathrm{g} / \mathrm{ml}$ ) or lysozyme (right; 3.9-125 $\mu \mathrm{g} /$ $\mathrm{ml}$ ). (B) Representative images showing effect of INH and lysozyme treatment on bacterial survival scored by spotting bacteria exposed to INH (left; $0.002-0.064 \mu \mathrm{g} / \mathrm{ml}$ ) or lysozyme (right; $3.9-125 \mu \mathrm{g} / \mathrm{ml}$ ) in the presence of $0.02 \%$ DMSO or $5 \mu \mathrm{M}$ C10-IMD on antibiotic-free $7 \mathrm{H} 10$ plates.

anti-tubercular agent and Rv3161c was proposed to convert pro-drug DAQ into its active form ${ }^{29}$. Despite all of these previous studies, the mechanism of action of the Rv3161c in response to antimicrobial agents is still unclear.

We discovered that $r v 3161 c$ is highly expressed in response to the C10-IMD compound that previously has been shown to potentiate INH sensitivity ${ }^{6}$. Induced $r v 3161 c$ expression was also observed as a response to 2 -aminoimidazoles (2-AI), a class of molecules that potentiate sensitivity to $\beta$-lactam antibiotics by increasing cell permeability ${ }^{14}$. Several studies have suggested that Rv3161c is involved in lipid metabolism and cell wall biogenesis ${ }^{11,17-19}$. In this study, we found that $\Delta r v 3161 \mathrm{c}$ mutants become more resistant to INH as well as lysozyme, an enzyme degrading the peptidoglycan layer of the cell wall (Fig. 5). We hypothesize that elevated Rv3161c may disturb the cell wall integrity and increase cell permeability, resulting in increased INH and lysozyme sensitivity. The $\Delta r v 3161 \mathrm{c}$ mutants can still be sensitized against both INH and lysozyme by C10-IMD, demonstrating that C10-IMD functions independent of Rv3161c to elicit these effects (Fig. 5). We tried to generate a $\Delta r v 3160 c$ mutant to test whether elevated Rv3161c in the absence of C10-IMD could increase sensitivity against INH and lysozyme, however we failed to generate such a mutant in several attempts.

In this study, we clarified the function of Rv3160c as a TetR-like transcriptional repressor that regulates $r v 3161 c$ expression. Despite the fact that several publications have suggested the $r v 3160 c-r v 3161 c$ operon to be involved in key processes such as drug detoxification, lipid metabolism and cell wall biogenesis ${ }^{11,14-19,29}$, the function of the Rv3161c remains unknown. Further investigation aiming to elucidate the function of Rv3161c will lead to important insights into $M t b$ biology.

\section{Methods}

Bacterial strains and growth conditions. WT E. coli DH5a was grown in Luria Broth (Difco) as shaking cultures at $37^{\circ} \mathrm{C}$ and kanamycin at $50 \mu \mathrm{g} / \mathrm{ml}$ was used for selection when required.

WT $M t b$ Erdman and the $r v 3161 c$ knockout with $M t b$ Erdman background were grown in either Middlebrook 7 H9 medium (BD Difco) supplemented with $0.05-0.1 \%$ Tween 80 and $10 \%$ ADS $(0.5 \%$ albumin, $0.2 \%$ dextrose and $0.085 \%$ saline) or Sauton's medium $\left[0.0005 \%\right.$ (w/v) $\mathrm{KH}_{2} \mathrm{PO}_{4}, 0.0005 \%(\mathrm{w} / \mathrm{v}) \mathrm{MgSO}_{4}, 0.004 \%$ (w/v) L-asparagine, $0.06 \%$ glycerol, $0.00005 \%(\mathrm{w} / \mathrm{v})$ ferric ammonium citrate, $0.002 \%(\mathrm{w} / \mathrm{v})$ citric acid, $0.0001 \% \mathrm{ZnSO}_{4}$, $\mathrm{pH} 7$ 7] supplemented with $0.1 \%$ Tween 80 as liquid shaking cultures at $37^{\circ} \mathrm{C}$. These strains were also grown on Middlebrook 7H10 solid medium (Remel) supplemented with $0.05 \%$ Tween $80,0.2-0.5 \%$ Glycerol and $10 \%$ ADS at $37^{\circ} \mathrm{C}$. Kanamycin at $20 \mu \mathrm{g} / \mathrm{ml}$ and anhydrotetracycline $(\mathrm{aTc})$ at $100 \mathrm{ng} / \mathrm{ml}$ were used when required. All the strains used in this work are listed in Table S1. 
Construction of rv3160c-CRISPRi and rv3161c-CRISPRi strains. To investigate regulation of the $r v 3160 c-r v 3161 c$ operon, we silenced expression of the $r v 3160 c$ and the $r v 3161 c$ genes individually by using the dCas $9_{\text {sth } 1}$ CRISPRi system ${ }^{30}$. This system utilizes a catalytically inactivated CRISPR1 cas 9 allele and provides an RNA-guided platform for sequence-specific control of gene expression. The single guide RNAs (sgRNAs) for each gene were determined and ranked by PAM score. Forward and reverse oligonucleotides (Table S2) corresponding to the top-ranked sgRNA sequence for each gene were annealed together to form double stranded DNA fragments and subsequently cloned into the BsmBI site of pLJR965 as described before ${ }^{30}$. The resulting plasmids, named pSA253 (targeting $r v 3160 c$ ) and pSA256 (targeting $r v 3161 c$ ), were electroporated into $M t b$ Erdman to obtain TB3 and TB4 respectively.

RNA isolation and cDNA synthesis. Mtb Erdman strains harboring pLRT965 (empty vector), pSA253 (targeting $r v 3160 c$ ) or pSA256 (targeting $r v 3161 c$ ) were grown in 7H9 medium supplemented with $0.05 \%$ Tween $80,10 \% \mathrm{ADS}$ and $20 \mu \mathrm{g} / \mathrm{ml}$ kanamycin until their optical density $\left(\mathrm{OD}_{600}\right)$ reaches to 0.3 in the presence of aTc $(100 \mathrm{ng} / \mathrm{ml})$ to induce expression of gene-specific sgRNA. Same strains were also cultivated similarly but in the absence of aTc as non-induced controls. Equivalents of $6 \mathrm{OD}_{600}$-unit cells were harvested by centrifugation and resuspended in $1 \mathrm{ml}$ TRI Reagent (Invitrogen). Cell suspensions were transferred into screw-cap homogenizing tubes containing $0.4 \mathrm{ml}$ of $0.1 \mathrm{~mm}$ Zirconia/Silica beads (BioSpec) and lysed in a bead beater 8 times for $30 \mathrm{~s}$ with a 1-min rest on ice between each cycle. After a centrifugation at 13,000 g for $45 \mathrm{~s}$, supernatants were transferred into new tubes and mixed with $300 \mu \mathrm{l}$ chloroform followed by incubation at room temperature for $2 \mathrm{~min}$. The samples were centrifuged at $13,000 \mathrm{~g}$ for $5 \mathrm{~min}$ and aqueous phase were transferred into new tubes. An equal volume of absolute ethanol was mixed with each sample and subsequently transferred into RNeasy columns (Qiagen) followed by purification according to the manufacturer's instruction. Isolated RNA samples were then DNase-treated with TURBO DNA-free kit (Invitrogen) to eliminate any genomic DNA contamination. cDNA was prepared using qScript cDNA Synthesis kit (Quantabio) according to the manufacturer's instruction.

Quantitative real-time PCR. Expression levels of $r v 3160 c$ and $r v 3161 c$ genes were determined by qRTPCR with cDNA equivalent to 50 ng RNA using Maxima SYBR Green/ROX qPCR Master Mix (Thermo Scientific) and the oligonucleotides listed in Table S3. RNA samples that were not reverse transcribed were used to confirm that there were no DNA contamination, and the integrity of the amplicons were confirmed by melting curves for each sample. All qRT-PCR experiments were performed with three technical replicates derived from three biological replicates. Signals were normalized to the housekeeping sigA mRNA and quantified by the Livak $\left(2^{-\Delta \Delta \mathrm{Ct}}\right)$ method $^{31}$. The $\mathrm{Ct}$ values of induced samples were compared to the corresponding $\mathrm{Ct}$ values of uninduced samples. Statistical analysis was performed using the one-tailed $t$-test and the results were plotted as bar graphs based on average and standard deviation obtained from three biological replicates by GraphPad Prism version 8.4.3 for Windows, GraphPad Software, San Diego, California USA, www.graphpad.com.

Electrophoretic mobility shift assay. EMSA was performed with $142 \mathrm{bp} r v 3161 \mathrm{c}$ upstream fragment (undigested or digested with BgII, HincII or TaqI), $86 \mathrm{bp}$ rv3161c upstream fragment (wild type or altered) or $204 \mathrm{bp} c f p 10$ upstream fragment. The $142 \mathrm{bp} r v 3161 c$ upstream sequence was directly amplified from chromosomal DNA with BS3161F and BS3161R oligonucleotides. Complementary oligonucleotides bearing the $86 \mathrm{bp}$ $r v 3161 c$ upstream sequence (wild type or altered) were annealed and amplified with UniPal_F and UniPal_R oligonucleotides (Table S4). cfp10 upstream sequence was also directly amplified from chromosomal DNA with EMSA_CFP10-F and EMSA_CFP10-R oligonucleotides (Table S4). The binding reactions were prepared in $10 \mathrm{mM}$ Tris- $\mathrm{HCl}$ (pH 8.0), $150 \mathrm{mM} \mathrm{KCl}, 0.5 \mathrm{mM}$ EDTA (pH 8.0), 0.1\% Triton-X 100, $12.5 \%$ glycerol, $0.2 \mathrm{mM}$ DTT, $0.1 \mathrm{mg} / \mathrm{ml} \mathrm{BSA}$ and $10 \mathrm{ng} / \mathrm{ul}$ Poly-dIdC in the presence of 1 or 4 pmol DNA targets, 10 or $56 \mathrm{pmol}$ Rv3160c (produced by the Protein Expertise Platform at Umeå University in 1× PBS) and/or $50 \mathrm{nmol} \mathrm{C10-IMD} \mathrm{and}$ incubated for $30 \mathrm{~min}$ at $37^{\circ} \mathrm{C}$. Reaction mixes were then loaded onto $6 \%$ polyacrylamide gels and run at $80 \mathrm{~V}$ in $0.5 \% \mathrm{TBE}$ buffer for $75 \mathrm{~min}$. The gels were then stained in $3 \times$ GelRed (Biotium) buffer for $15 \mathrm{~min}$ followed by washing with MQ water for 15 min twice. The DNA was visualized with UV-transilluminator and signals were analyzed by Image J Software version $1.52 \mathrm{a}^{32}$.

Fluorescence spectroscopy. The interaction between C10-IMD and Rv3160c was analyzed by concentration-dependent response of C10-IMD on Rv3160c tryptophan fluorescence emission measured in Hitachi F-4500 fluorescence spectrophotometer. Rv3160c emission spectra were acquired using $10 \mu \mathrm{M}$ of protein dissolved in $1 \times \mathrm{PBS}\left(\mathrm{pH} 6.8\right.$ ) with or without adding C10-IMD and was incubated for $1 \mathrm{~min}$ at $37^{\circ} \mathrm{C}$ in a quartz cuvette. The resulting solution was excited at $290 \mathrm{~nm}$ for tryptophan residue and the intrinsic fluorescence emission was scanned from 300 to $460 \mathrm{~nm}$. The data from each read was then combined and plotted using the Spectragryph Software version 1.2.13 $3^{33}$. The fluorescence quenching data have been corrected for inner filter effects. Fluorescence quenching was determined by analyzing the data by the classical Stern-Volmer equation:

$$
\mathrm{F}_{0} / \mathrm{F}=1+k_{\mathrm{q}} \tau_{0}[\mathrm{Q}]=1+K_{\mathrm{sv}}[\mathrm{Q}]
$$

where $\mathrm{F}_{\mathrm{o}}$ and $\mathrm{F}$ are the fluorescence intensities in the absence and presence of the quencher, respectively. $\mathrm{K}_{\mathrm{q}}$ is the bimolecular quenching rate constant and $\mathrm{K}_{\mathrm{sv}}$ is the dynamic quenching constant. Average lifetime of the protein in absence of the quencher is expressed in terms of $\tau_{o}$ whereas $[Q]$ is the concentration of the free quencher. When small molecules interact with several comparable sites in a protein, the equilibrium between liberated and bound molecules is expressed by the equation: 


$$
\begin{aligned}
\log \left(\left(\mathrm{F}_{0}-\mathrm{F}\right) / \mathrm{F}\right) & =\log K_{\mathrm{b}}+\mathrm{n} \log [\mathrm{Q}] \\
\mathrm{K}_{\mathrm{D}} & =1 / K_{\mathrm{b}}
\end{aligned}
$$

where $\mathrm{K}_{\mathrm{b}}$ represents binding constant for quencher-protein interaction, $\mathrm{n}$ is the number of binding sites per molecule of Rv3160c, $\mathrm{K}_{\mathrm{D}}$ denotes binding dissociation constant.

Circular dichroism (CD) spectroscopy. CD spectroscopy was performed with Rv3160c as described earlier ${ }^{34}$. The native secondary structure of Rv3160c was compared using far-UV range CD spectra in presence of various concentrations of C10-IMD $(10 \mu \mathrm{M}$ and $100 \mu \mathrm{M})$ and $86 \mathrm{bp} r v 3161 \mathrm{c}$ upstream DNA bait $(1 \mu \mathrm{M}$ and $10 \mu \mathrm{M})$. The spectra were recorded using Jasco CD spectrometer at a range of 200 to $260 \mathrm{~nm}$ from $10 \mu \mathrm{M}$ of $\mathrm{Rv} 3160 \mathrm{c}$ in optically clear buffer under the nitrogen atmosphere at $25^{\circ} \mathrm{C}$. The buffer baseline corrected spectra were processed with an average of 5 scans and presented as units of mean residue ellipticity.

Impact of isoniazid and lysozyme on bacterial growth and survival. The bactericidal/bacteriostatic properties of isoniazid and lysozyme were assayed at varying concentrations $(0.002-0.064 \mu \mathrm{g} / \mathrm{ml}$ for INH or $3.9-125 \mu \mathrm{g} / \mathrm{ml}$ for lysozyme) in Sauton's medium with $0.02 \%$ DMSO or $5 \mu$ M C10-IMD for both Mtb Erdman wild type and $\Delta r v 3161 \mathrm{c}$ strains as described in our previous study ${ }^{35}$.

Received: 7 August 2020; Accepted: 31 December 2020

Published online: 15 January 2021

\section{References}

1. WHO. Global tuberculosis report 2019. (2019).

2. Bloemberg, G. V. et al. Acquired resistance to bedaquiline and delamanid in therapy for tuberculosis. N. Engl. J. Med. 373, 1986-1988. https://doi.org/10.1056/NEJMc1505196 (2015).

3. Hoffmann, H. et al. Delamanid and bedaquiline resistance in Mycobacterium tuberculosis ancestral Beijing genotype causing extensively drug-resistant tuberculosis in a tibetan refugee. Am. J. Respir. Crit. Care Med. 193, 337-340. https://doi.org/10.1164/ rccm.201502-0372LE (2016).

4. Polsfuss, S. et al. Emergence of low-level delamanid and bedaquiline resistance during extremely drug-resistant tuberculosis treatment. Clin. Infect. Dis. 69, 1229-1231. https://doi.org/10.1093/cid/ciz074 (2019).

5. Somoskovi, A., Bruderer, V., Homke, R., Bloemberg, G. V. \& Bottger, E. C. A mutation associated with clofazimine and bedaquiline cross-resistance in MDR-TB following bedaquiline treatment. Eur. Respir. J. 45, 554-557. https://doi.org/10.1183/09031936.00142 914 (2015).

6. Flentie, K. et al. Chemical disarming of isoniazid resistance in Mycobacterium tuberculosis. Proc. Natl. Acad. Sci. U.S.A. 116, 10510-10517. https://doi.org/10.1073/pnas.1818009116 (2019).

7. Sukheja, P. et al. A novel small-molecule inhibitor of the Mycobacterium tuberculosis demethylmenaquinone methyltransferase meng is bactericidal to both growing and nutritionally deprived persister cells. $m$ Bio https://doi.org/10.1128/mBio.02022-16 (2017).

8. Vilcheze, C. et al. Enhanced respiration prevents drug tolerance and drug resistance in Mycobacterium tuberculosis. Proc. Natl. Acad. Sci. U.S.A. 114, 4495-4500. https://doi.org/10.1073/pnas.1704376114 (2017).

9. Karp, P. D. et al. The BioCyc collection of microbial genomes and metabolic pathways. Brief Bioinform. 20, 1085-1093. https://doi. org/10.1093/bib/bbx085 (2019).

10. Romero, P. R. \& Karp, P. D. Using functional and organizational information to improve genome-wide computational prediction of transcription units on pathway-genome databases. Bioinformatics 20, 709-717. https://doi.org/10.1093/bioinformatics/btg471 (2004)

11. Betts, J. C. et al. Signature gene expression profiles discriminate between isoniazid-, thiolactomycin-, and triclosan-treated $M y c o-$ bacterium tuberculosis. Antimicrob. Agents Chemother. 47, 2903-2913. https://doi.org/10.1128/aac.47.9.2903-2913.2003 (2003).

12. Parikh, S. L., Xiao, G. \& Tonge, P. J. Inhibition of InhA, the enoyl reductase from Mycobacterium tuberculosis, by triclosan and isoniazid. Biochemistry 39, 7645-7650. https://doi.org/10.1021/bi0008940 (2000).

13. Balhana, R. J., Singla, A., Sikder, M. H., Withers, M. \& Kendall, S. L. Global analyses of TetR family transcriptional regulators in mycobacteria indicates conservation across species and diversity in regulated functions. BMC Genom. 16, 479. https://doi. org/10.1186/s12864-015-1696-9 (2015).

14. Jeon, A. B. et al. 2-aminoimidazoles potentiate ss-lactam antimicrobial activity against Mycobacterium tuberculosis by reducing sslactamase secretion and increasing cell envelope permeability. PLoS ONE 12, e0180925. https://doi.org/10.1371/journal.pone.01809 25 (2017).

15. Waddell, S. J. et al. The use of microarray analysis to determine the gene expression profiles of Mycobacterium tuberculosis in response to anti-bacterial compounds. Tuberculosis (Edinb) 84, 263-274. https://doi.org/10.1016/j.tube.2003.12.005 (2004).

16. Dutta, N. K., Mazumdar, K., Dastidar, S. G., Karakousis, P. C. \& Amaral, L. New patentable use of an old neuroleptic compound thioridazine to combat tuberculosis: a gene regulation perspective. Recent Pat Antiinfect. Drug Discov. 6, 128-138. https://doi. org/10.2174/157489111796064597 (2011).

17. Dutta, N. K., Mehra, S. \& Kaushal, D. A Mycobacterium tuberculosis sigma factor network responds to cell-envelope damage by the promising anti-mycobacterial thioridazine. PLoS ONE 5, e10069. https://doi.org/10.1371/journal.pone.0010069 (2010).

18. Aguilar-Ayala, D. A. et al. The transcriptome of Mycobacterium tuberculosis in a lipid-rich dormancy model through RNAseq analysis. Sci. Rep. 7, 17665. https://doi.org/10.1038/s41598-017-17751-x (2017).

19. Boshoff, H. I. et al. The transcriptional responses of Mycobacterium tuberculosis to inhibitors of metabolism: novel insights into drug mechanisms of action. J. Biol. Chem. 279, 40174-40184. https://doi.org/10.1074/jbc.M406796200 (2004).

20. Cortes, T. et al. Genome-wide mapping of transcriptional start sites defines an extensive leaderless transcriptome in Mycobacterium tuberculosis. Cell. Rep. 5, 1121-1131. https://doi.org/10.1016/j.celrep.2013.10.031 (2013).

21. Samworth, C. M., Degli Esposti, M. \& Lenaz, G. Quenching of the intrinsic tryptophan fluorescence of mitochondrial ubiquinolcytochrome-c reductase by the binding of ubiquinone. Eur. J. Biochem. 171, 81-86. https://doi.org/10.1111/j.1432-1033.1988.tb137 61.x (1988).

22. Hinrichs, W. et al. Structure of the Tet repressor-tetracycline complex and regulation of antibiotic resistance. Science 264, 418-420. https://doi.org/10.1126/science.8153629 (1994). 
23. Kisker, C., Hinrichs, W., Tovar, K., Hillen, W. \& Saenger, W. The complex formed between Tet repressor and tetracycline-Mg2+ reveals mechanism of antibiotic resistance. J. Mol. Biol. 247, 260-280. https://doi.org/10.1006/jmbi.1994.0138 (1995).

24. Orth, P. et al. Conformational changes of the Tet repressor induced by tetracycline trapping. J. Mol. Biol. 279, 439-447. https:// doi.org/10.1006/jmbi.1998.1775 (1998).

25. Orth, P., Schnappinger, D., Hillen, W., Saenger, W. \& Hinrichs, W. Structural basis of gene regulation by the tetracycline inducible Tet repressor-operator system. Nat. Struct. Biol. 7, 215-219. https://doi.org/10.1038/73324 (2000).

26. Berens, C. \& Hillen, W. Gene regulation by tetracyclines. Constraints of resistance regulation in bacteria shape TetR for application in eukaryotes. Eur. J. Biochem. 270, 3109-3121. https://doi.org/10.1046/j.1432-1033.2003.03694.x (2003).

27. Saenger, W., Orth, P., Kisker, C., Hillen, W. \& Hinrichs, W. The tetracycline repressor-a paradigm for a biological switch. Angew. Chem. Int. Ed. Engl. 39, 2042-2052. https://doi.org/10.1002/1521-3773(20000616)39:12\%3c2042::aid-anie2042\%3e3.0.co;2-c (2000).

28. Gomez, A., Andreu, N., Ferrer-Navarro, M., Yero, D. \& Gibert, I. Triclosan-induced genes $R v 1686 c-R v 1687 c$ and $R v 3161 c$ are not involved in triclosan resistance in Mycobacterium tuberculosis. Sci. Rep. 6, 26221. https://doi.org/10.1038/srep26221 (2016).

29. Odingo, J. et al. Synthesis and evaluation of the 2,4-diaminoquinazoline series as anti-tubercular agents. Bioorg. Med. Chem. 22, 6965-6979. https://doi.org/10.1016/j.bmc.2014.10.007 (2014).

30. Rock, J. M. et al. Programmable transcriptional repression in mycobacteria using an orthogonal CRISPR interference platform. Nat. Microbiol. 2, 16274. https://doi.org/10.1038/nmicrobiol.2016.274 (2017).

31. Livak, K. J. \& Schmittgen, T. D. Analysis of relative gene expression data using real-time quantitative PCR and the 2(-Delta Delta C(T)) Method. Methods 25, 402-408. https://doi.org/10.1006/meth.2001.1262 (2001).

32. Rasband, W. S. ImageJ, U. S. National Institutes of Health, Bethesda, Maryland, USA, https://imagej.nih.gov/ij/ (1997-2018).

33. Menges, F. "Spectragryph - optical spectroscopy software", Version 1.2.9. http://www.effemm2.de/spectragryph/ (2018).

34. Sarkar, S. et al. Ellagic acid, a dietary polyphenol, inhibits tautomerase activity of human macrophage migration inhibitory factor and its pro-inflammatory responses in human peripheral blood mononuclear cells. J. Agric. Food Chem. 63, 4988-4998. https:// doi.org/10.1021/acs.jafc.5b00921 (2015).

35. Tukenmez, H. et al. Mycobacterium tuberculosis virulence inhibitors discovered by Mycobacterium marinum high-throughput screening. Sci. Rep. 9, 26. https://doi.org/10.1038/s41598-018-37176-4 (2019).

\section{Acknowledgements}

This project has been supported under the framework of the JPIAMR - Joint Programming Initiative on Antimicrobial Resistance. C.L. is supported by the Kempe Foundation [SMK-1648] and the Åke Wilton's Fund [FS 2.1.6-1577-17]. F.A. and C.L.S. are supported by the National Institutes of Health Grant [R01 AI134847] and the Erling-Persson Family Foundation [P20-00473]. F.A. is supported by the Swedish Research Council (JPIAMR) [2018-00969] and the Kempe Foundation [SMK-1755]. C.L.S. is supported by the Burroughs Welcome Fund Investigators in the Pathogenesis of Infectious Disease [1016717]. G.A.H. is supported by the National Science Foundation Graduate Research Fellowship [DGE-1745038] and the National Institute of General Medical Sciences Cell and Molecular Biology Training Grant [GM007067]. The personnel of the Protein Expertise Platform $(\mathrm{PEP})$ at the Chemical Biological Center (KBC) at Umeå University is acknowledged for their skills and support in protein purification.

\section{Author contributions}

C.L., H.T., S.S. and S.A. designed the experiments. H.T., C.L., S.S., S.A., A.K. and I.E. performed the experiments. C.L., H.T., S.S., S.A., A.K., I.E., G.A.H., C.L.S. and F.A. analyzed the data. H.T., C.L., S.S. and S.A. wrote the main manuscript text. All authors reviewed the manuscript and the revisions.

\section{Funding}

Open Access funding provided by Umea University.

\section{Competing interests}

C.L.S. and F.A. have ownership interests in Quretech Bio AB, which licences C10-IMD. The rest of the authors have no competing interest.

\section{Additional information}

Supplementary Information The online version contains supplementary material available at https://doi. org/10.1038/s41598-021-81104-y.

Correspondence and requests for materials should be addressed to H.T. or C.L.

Reprints and permissions information is available at www.nature.com/reprints.

Publisher's note Springer Nature remains neutral with regard to jurisdictional claims in published maps and institutional affiliations.

\footnotetext{
(c) (i) Open Access This article is licensed under a Creative Commons Attribution 4.0 International License, which permits use, sharing, adaptation, distribution and reproduction in any medium or format, as long as you give appropriate credit to the original author(s) and the source, provide a link to the Creative Commons licence, and indicate if changes were made. The images or other third party material in this article are included in the article's Creative Commons licence, unless indicated otherwise in a credit line to the material. If material is not included in the article's Creative Commons licence and your intended use is not permitted by statutory regulation or exceeds the permitted use, you will need to obtain permission directly from the copyright holder. To view a copy of this licence, visit http://creativecommons.org/licenses/by/4.0/.
}

(C) The Author(s) 2021 